STRUCTURAL BIOLOGY COMMUNICATIONS

ISSN 2053-230X

Received 11 December 2019

Accepted 27 January 2020

Edited by F. T. Tsai, Baylor College of Medicine, Houston, USA

Keywords: FtsZ; cytokinesis; treadmilling; protofilaments; Z-ring.

PDB references: E. coli FtsZ(L178E), complex with GDP, 6umk; complex with GTP, 6unx

Supporting information: this article has supporting information at journals.iucr.org/f

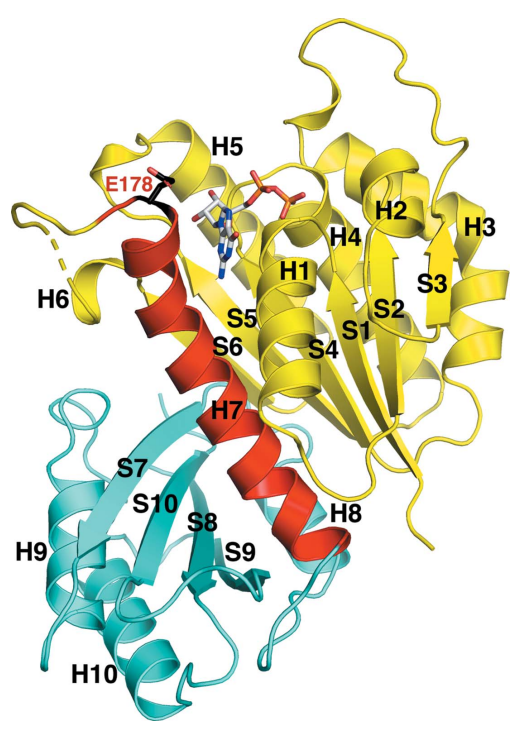

OPEN $\odot$ ACCESS

\section{High-resolution crystal structures of Escherichia coli FtsZ bound to GDP and GTP}

\author{
Maria A. Schumacher, ${ }^{a *}$ Tomoo Ohashi, $^{\mathrm{b}}{\text { Lauren } \text { Corbin }^{\mathrm{c}} \text { and Harold P. Erickson }}^{\mathrm{a}, \mathrm{b}}$ \\ a Department of Biochemistry, Duke University School of Medicine, Box 3711, DUMC, Durham, NC 27710, USA, \\ ${ }^{\mathbf{b}}$ Department of Cell Biology, Duke University School of Medicine, Box 3711, DUMC, Durham, NC 27710, USA, and \\ ${ }^{\mathrm{c}}$ Department of Biomedical Engineering, Duke University, Durham, NC 27708, USA. *Correspondence e-mail: \\ maria.schumacher@duke.edu
}

Bacterial cytokinesis is mediated by the Z-ring, which is formed by the prokaryotic tubulin homolog FtsZ. Recent data indicate that the Z-ring is composed of small patches of FtsZ protofilaments that travel around the bacterial cell by treadmilling. Treadmilling involves a switch from a relaxed (R) state, favored for monomers, to a tense (T) conformation, which is favored upon association into filaments. The $\mathrm{R}$ conformation has been observed in numerous monomeric FtsZ crystal structures and the T conformation in Staphylococcus aureus FtsZ crystallized as assembled filaments. However, while Escherichia coli has served as a main model system for the study of the Z-ring and the associated divisome, a structure has not yet been reported for E. coli FtsZ. To address this gap, structures were determined of the E. coli FtsZ mutant FtsZ(L178E) with GDP and GTP bound to 1.35 and $1.40 \AA$ resolution, respectively. The E. coli FtsZ(L178E) structures both crystallized as straight filaments with subunits in the $\mathrm{R}$ conformation. These high-resolution structures can be employed to facilitate experimental cell-division studies and their interpretation in E. coli.

\section{Introduction}

Cytokinesis, the process by which a cell divides into daughter cells, is essential for all life. Bacteria accomplish this task by employing a multiprotein complex called the divisome. Work over the past three decades has witnessed the discovery and characterization of most of the divisome proteins in Escherichia coli (Margolin, 2005; Adams \& Errington, 2009; Rowlett \& Margolin, 2015; Tsang \& Bernhardt, 2015; Du \& Lutkenhaus, 2017; den Blaauwen et al., 2017; Lutkenhaus et al., 2017; Söderström \& Daley, 2017). FtsZ, a bacterial homolog of tubulin, assembles onto a ring-like scaffold called the Z-ring, which serves as the foundation for cell division by the divisome (Bi \& Lutkenhaus, 1991; Nogales et al., 1998; Erickson et al., 2010; Haeusser \& Margolin, 2016; Du \& Lutkenhaus, 2019). FtsZ assembles into single-stranded protofilaments, and these associate into clusters that are anchored to the cell membrane via a disordered linker and a C-terminal peptide that attaches to FtsA, ZipA and/or other proteins (Ma et al., 1996; Pichoff \& Lutkenhaus, 2005; Gardner et al., 2013; Buske \& Levin, 2013; Sundararajan et al., 2015). Recent work has shown that clusters of membrane-bound FtsZ move around the circumference of the cell by treadmilling, and that these moving patches carry with them the divisome cell-wall remodeling machinery (Strauss et al., 2012; Rowlett \& Margolin, 2014; Bisson-Filho et al., 2017; Yang et al., 2017; Perez et al., 2019; Li et al., 2018; Söderström et al., 2019). FtsZ treadmilling has also been visualized in vitro on supported lipid bilayers (Loose \& Mitchison, 2014; Ramirez-Diaz et al., 2018). 
Treadmilling is now established as the primary mechanism for FtsZ assembly dynamics.

Apart from treadmilling at steady state, FtsZ assembly in vitro exhibits two aspects of cooperative assembly: an unfavorable nucleation step and a critical concentration. However, this raises the question of how a single-stranded protofilament with only one type of bond can exhibit cooperative assembly. Several groups have suggested that this could be achieved if FtsZ had two conformations, one with a high and one with a low affinity for making longitudinal bonds (Michie \& Löwe, 2006; Dajkovic \& Lutkenhaus, 2006; Dajkovic et al., 2008; Huecas et al., 2008; Miraldi et al., 2008). To explain the cooperativity, the model proposes that FtsZ monomers are highly favored to be in the low-affinity conformation, whereas assembly into a protofilament switches the subunit to favor the high-affinity conformation (Michie \& Löwe, 2006; Dajkovic \& Lutkenhaus, 2006; Dajkovic et al., 2008; Huecas et al., 2008; Miraldi et al., 2008).

Prior to 2012, numerous FtsZ crystal structures were solved from different bacterial species in GTP-bound, GDP-bound or apo states (Löwe \& Amos, 1998; Leung et al., 2000; Cordell et al., 2003; Oliva et al., 2004, 2007). These structures all had the same basic structure: two globular subdomains connected by a long helix, H7. The N-terminal subdomain contains the binding site for GTP, and the C-terminal subdomain has, by analogy with tubulin protofilaments, key catalytic residues that contact the GTP in the subunit below to initiate GTP hydrolysis (Nogales et al., 1998; Erickson, 1998; Scheffers et al., 2002). Importantly, these structures were mostly of FtsZ monomers.

A major advance in our understanding of FtsZ structure and polymerization was achieved when two laboratories independently crystallized FtsZ from Staphylococcus aureus (SaFtsZ) and found that it crystallized in the form of long, straight protofilaments (Matsui et al., 2012; Elsen et al., 2012). The FtsZ in these protofilaments showed a striking conformational change relative to the previous monomeric forms, even the closely related Bacillus subtilis FtsZ (BsFtsZ) (Oliva et al., 2007; Matsui et al., 2012; Elsen et al., 2012). As noted, the globular domain of both FtsZ and tubulin is divided into two subdomains. The conformational change from BsFtsZ to SaFtsZ involved a large rotation $\left(25-28^{\circ}\right)$ of the C-terminal subdomain relative to the N-terminal subdomain. Accompanying this rotation, the helix $\mathrm{H} 7$ shifted downwards by a turn (Matsui et al., 2012; Elsen et al., 2012). This new structure of SaFtsZ assembled in straight protofilaments with a large interface and was an excellent candidate for the high-affinity, protofilament-forming conformation, while the monomeric BsFtsZ represented the low-affinity conformation. The two conformations of FtsZ have been designated $\mathrm{T}$ (tense) for the high-affinity state and R (relaxed) for the low-affinity state (Matsui et al., 2014; Fujita et al., 2017). Alternatively, the two conformations have been designated open and closed, referring to the cleft between the two subdomains (Wagstaff et al., 2017). We will adopt the $R$ and $T$ nomenclature here.

Although wild-type SaFtsZ assembles preferentially into protofilaments with subunits in the $\mathrm{T}$ conformation, several mutants of SaFtsZ have been found assembled into protofilaments with subunits in the $\mathrm{R}$ conformation (Matsui et al., 2014; Wagstaff et al., 2017). Interestingly, Fujita and coworkers obtained a crystal of wild-type SaFtsZ containing subunits in both R and T states (Fujita et al., 2017). Apparently, there is only a small free-energy difference between the $\mathrm{R}$ and $\mathrm{T}$ conformations when SaFtsZ subunits are assembled into protofilaments under crystallization conditions.

Strikingly, although $E$. coli has provided the most detailed understanding of the machinery and mechanism of bacterial cell division, the structure of the E. coli FtsZ (EcFtsZ) protein has remained elusive. A likely problem is the tendency of EcFtsZ to aggregate under non-assembly conditions (Mukherjee et al., 1993). Du and coworkers provided a potentially important advance when they explored several EcFtsZ mutants that had previously been identified as defective in assembly (Du et al., 2015; Li et al., 2013). Du and coworkers focused on one mutant, L178E, which they confirmed to be assembly-incompetent in the presence of GTP. They also found that EcFtsZ(L178E) lost its tendency to aggregate when bound to GDP, as determined by sizeexclusion chromatography (Du et al., 2015). This suggested that this mutant would be a good candidate for crystallography, and indeed they reported that 'FtsZ-L178E is selfinteracting at higher concentrations since it assembled into protofilaments when crystallized' (Du et al., 2015). However, these crystals were apparently not suitable for effective structure determination; the structure was never described nor was it deposited in the Protein Data Bank (Joe Lutkenhaus, personal communication). Thus, we decided to address this gap and repeated crystallographic screens in order to obtain better crystals. Here, we report the 1.35 and $1.40 \AA$ resolution structures of E. coli FtsZ(L178E) bound to GDP and GTP, respectively.

\section{Experimental}

\subsection{Production and purification of $E$. coli FtsZ(L178E)}

The pET-11 vector encoding the $E$. coli $\mathrm{K}-12$ fts $Z$ gene was originally obtained from Bramhill and Thompson (Bramhill \& Thompson, 1994). Truncation and site-directed mutants were generated from the vector and were also expressed in pET-11. The E. coli FtsZ truncation mutant used in this study encodes residues $10-316$ of the protein. Mutants, fts $Z(L 178 E)$, $f t s Z(L 178 E / M 206 E)$ and $f t s Z(L 178 E / L 272 E)$, were also generated using QuikChange (Agilent) in the context of the truncated protein. E. coli BL21(DE3) cells were transformed with these expression vectors. Cells expressing each mutant protein were grown to an $\mathrm{OD}_{600}$ of $\sim 1.2$ and induced by adding $0.5 \mathrm{~m} M$ isopropyl $\beta$-D-1-thiogalatopyranoside for $3 \mathrm{~h}$ at $37^{\circ} \mathrm{C}$. After induction, the cells were centrifuged for $45 \mathrm{~min}$ at $15000 \mathrm{~g}$. The resultant cell pellets were frozen at $-80^{\circ} \mathrm{C}$ until use. Frozen cells were suspended in resuspension buffer consisting of $50 \mathrm{~m} M$ Tris- $\mathrm{HCl} \mathrm{pH} 7.9,100 \mathrm{~m} M \mathrm{NaCl}, 2 \mathrm{~m} M$ phenylmethylsulfonyl fluoride, and $0.75 \mathrm{mg} \mathrm{ml}^{-1}$ lysozyme was added. After $30 \mathrm{~min}$ on ice the cells were refrozen, thawed 
Table 1

Crystallization conditions for EcFtsZ(L178E)-nucleotide structures.

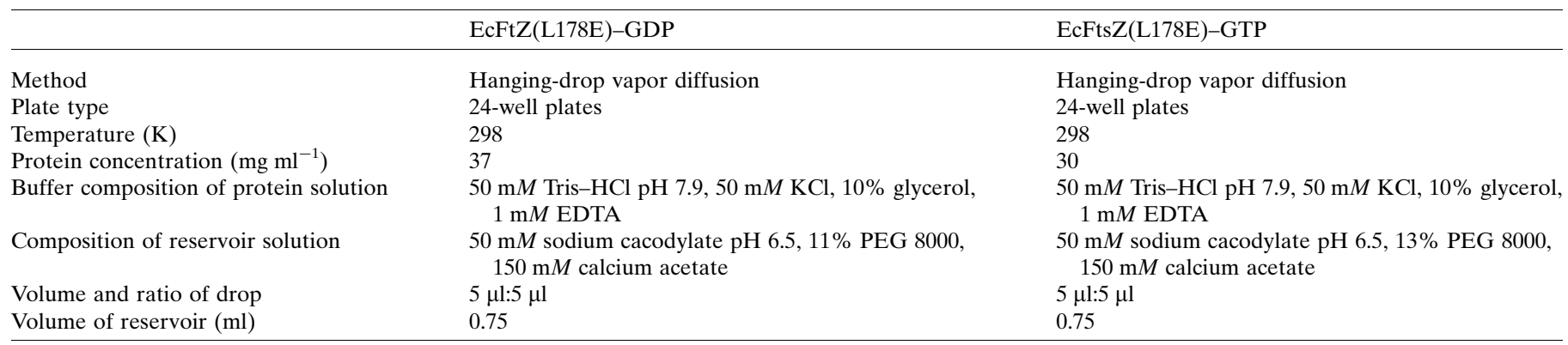

and lysed by sonication. The cells were sonicated eight times for $30 \mathrm{~s}$ on ice. The lysate was centrifuged at $80000 \mathrm{~g}$ for $30 \mathrm{~min}$ and the supernatant was recovered. The soluble bacterial proteins were first precipitated with $50 \%$ saturated ammonium sulfate. The resuspended proteins were then dialyzed against a buffer composed of $50 \mathrm{~m} M$ Tris- $\mathrm{HCl} \mathrm{pH}$ 7.9, $50 \mathrm{~m} M \mathrm{KCl}, 10 \%(v / v)$ glycerol, $1 \mathrm{~m} M$ EDTA. The proteins were purified by chromatography on a Resource Q anionexchange column (GE Healthcare) with a linear gradient from 50 to $500 \mathrm{~m} M \mathrm{KCl}$. Fractions containing protein were identified by SDS-PAGE analysis, and those containing protein were pooled and the protein was concentrated using a Microcon $30 \mathrm{kDa}$ cutoff concentrator (Millipore). The FtsZ protein concentrations were determined as described previously using bicinchoninic acid colorimetric assays ( $\mathrm{Lu}$ et al., 1998).

\subsection{Crystallization of $E$. coli FtsZ(L178E)-GDP and E. coli FtsZ(L178E)-GTP complexes}

Full-length, truncated and truncated mutant FtsZ proteins were used in crystallization trials. The EcFtsZ(L178E) protein truncated to the globular domain from residues 10 to 316 produced well diffracting crystals and thus was used for structural studies [and will be referred to here as EcFtsZ(L178E)]. To obtain crystals of EcFtsZ(L178E) with GDP, the protein was concentrated to $37 \mathrm{mg} \mathrm{ml}^{-1}$ and GDP was added to the solution, resulting in a final concentration of $1 \mathrm{~m} M$. The mixture was centrifuged for $3 \mathrm{~s}$ at $20000 \mathrm{~g}$ and then used in hanging-drop vapor-diffusion crystallization screens. The screens employed were Wizard Classic 1-4 (Rigaku), Wizard Cryo 1 and Cryo 2 (Rigaku), PEGRx 1 and PEGRx 2 (Hampton Research), Crystal Screen (Hampton Research), SaltRx 1 and SaltRx 2 (Hampton Research). The screens were all performed at room temperature using 24-well plates. In the screens, $1 \mu$ l protein solution was mixed with $1 \mu$ cystallization reagent and placed over the reservoir. The most promising crystals were obtained with Wizard Classic 3 condition 35 . This condition was further optimized and the best crystals were obtained by combining $5 \mu \mathrm{l}$ EcFtsZ(L178E)-GDP complex with $5 \mu \mathrm{l}$ crystallization solution consisting of $50 \mathrm{~m} M$ sodium cacodylate $\mathrm{pH}$ 6.5, 11\%(w/v) PEG 8000, $150 \mathrm{~m} M$ calcium acetate, $20 \%(v / v)$ glycerol and hanging the drop over a reservoir containing $750 \mu \mathrm{l}$ crystallization solution (Table 1). The crystals grew overnight and reached a maximum size of
$0.3 \times 0.5 \times 0.8 \mathrm{~mm}$ over $1-1.5$ weeks. The EcFtsZ(L178E)GTP crystals were obtained similarly using protein at $30 \mathrm{mg} \mathrm{ml}^{-1}$ with a final concentration of $1 \mathrm{~m} M$ GTP (Table 1). These crystals were isomorphous to the EcFtsZ(L678E)-GDP crystals. The EcFtsZ(L178E)-GTP complex was screened (at room temperature) using all of the commercial screens used for the EcFtsZ(L178E)-GDP complex. The best crystals of the EcFtsZ(L178E)-GTP complex were also obtained with Wizard Classic 3 condition 35. Optimal crystals were obtained by mixing the complex in a 1:1 ratio with a reservoir consisting of $13 \%(w / v)$ PEG $8000,50 \mathrm{~m} M$ sodium cacodylate $\mathrm{pH} 6.5$, $150 \mathrm{~m} M$ calcium acetate, $20 \%(v / v)$ glycerol and hanging the drop over a reservoir containing $750 \mu \mathrm{l}$ crystallization reagent (Table 1). The crystals grew overnight and reached similar sizes to the EcFtsZ(L178E)-GDP crystals in 1-2 weeks. Both crystals were cryopreserved directly from the crystallization solution, which acted as a cryosolvent. Data were collected on beamline 8.3.1 at the Advanced Light Source (ALS), USA. The crystals were looped and placed directly in the liquidnitrogen stream in the hutch and data were collected.

\subsection{Crystal structure determination of the $E$. coli FtsZ(L178E)-GDP and E. coli FtsZ(L178E)-GTP complexes}

X-ray intensity diffraction data for the EcFtsZ(L178E)GDP and EcFtsZ(L178E)-GTP complexes were collected on beamline 8.3.1 at the ALS. The data were processed using MOSFLM and scaled with SCALA (Leslie, 2006; Potterton et al., 2003; Table 2). Both the EcFtsZ(L178E)-GDP and EcFtsZ(L178E)-GTP crystals belonged to the monoclinic space group $P 2_{1}$. Molecular replacement (MR) using the Pseudomonas aeruginosa FtsZ structure (PDB entry 2vaw; Oliva et al., 2007) without the GDP and waters as a model was successful in solving the EcFtsZ(L178E)-GDP structure using MOLREP and Phaser (Vagin \& Teplyakov, 2010; McCoy et al., 2007). Both produced clear solutions [with a log likelihood gain (LLG) of 309.67 in Phaser]. There is one EcFtsZ(L178E) protein molecule in the crystallographic asymmetric unit. After an initial round of refinement in Phenix (Liebschner et al., 2019) the residues that were not identical between $P$. aeruginosa and $E$. coli were replaced by their $E$. coli counterparts. This structure was then subjected to multiple iterative cycles of rebuilding in $O$ and refinement in Phenix (Jones et al., 1991; Liebschner et al., 2019). GDP and water molecules were added in the final stages of refinement. The 
final model included EcFtsZ(L178E) residues 10-65, 67-169 and 173-316, one GDP molecule and 339 water molecules. Residues 63-73, 166-176 and 301-303 harbored weak electron density. The EcFtsZ(L178E)-GTP data were isomorphous with the EcFtsZL178E)-GDP data. However, in order to ensure an optimal starting model, MR was used with the EcFtsZ(L178E)-GDP structure minus the GDP as a search model in Phaser, which resulted in a solution with an LLG of 15 026. The final EcFtsZ(L178E)-GTP model included EcFtsZ(L178E) residues 10-169 and 173-316, one GTP molecule and 339 water molecules. Similar to the EcFtsZ(L178E)-GDP structure, weak electron density was observed for residues $62-73,165-176$ and $301-303$ in this structure. See Tables 2 and 3 for data-collection and refinement statistics.

\section{Results and discussion}

\subsection{E. coli FtsZ(L178E)-GDP and E. coli FtsZ(L178E)-GTP} structure determination

The EcFtsZ(L178E) protein, which previous studies had indicated to be a monomer in the presence of GDP or GTP (Du et al., 2015), produced well diffracting crystals in the presence of GDP and, when optimized, permitted us to collect

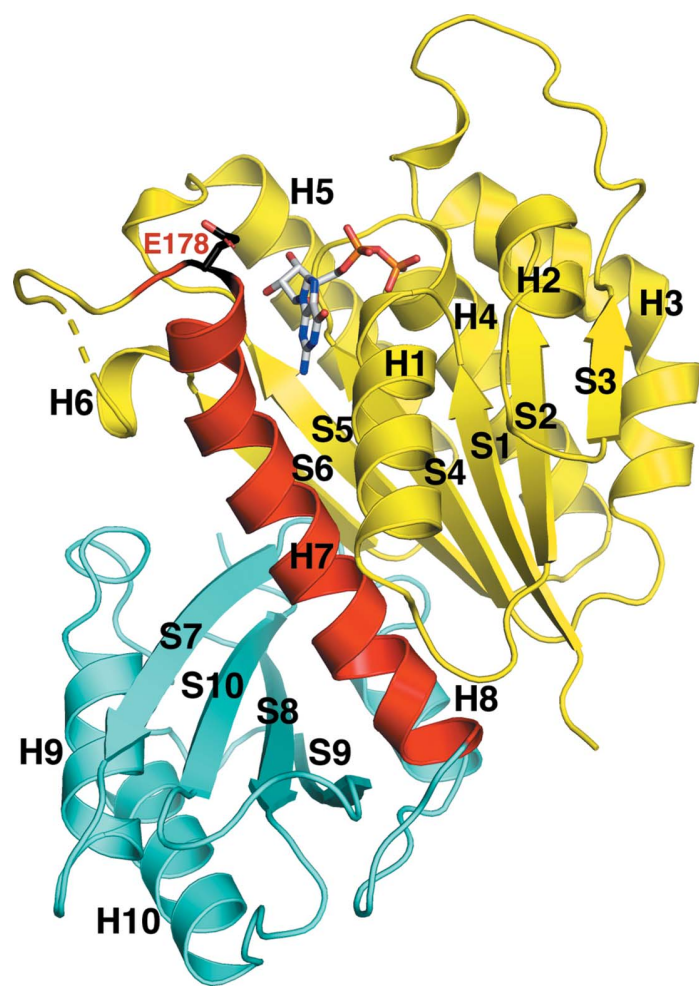

Figure 1

Overall structure of the EcFtsZ(L178E)-GDP complex. The protein is shown as a ribbon diagram with the $\mathrm{N}$-terminal subdomain (residues 9-178) colored yellow, the connecting $\mathrm{H} 7$ helix colored red and the C-terminal subdomain (residues 201-216) colored cyan. S and H indicate strands and helices, respectively. The bound GDP is shown as sticks. Also shown as sticks is residue Glu178, the site of the mutation. The structure is labeled according to the common nomenclature for the tubulin/FtsZ family of GTPases (Nogales et al., 1998). All ribbon diagrams were made using PyMOL (DeLano, 2002).
Table 2

Data collection and processing.

Values in parentheses are for the highest resolution shell.

\begin{tabular}{lll}
\hline & EcFtsZ(L178E)-GDP & EcFtsZ(L178E)-GTP \\
\hline Source & Beamline 8.3.1, ALS & Beamline 8.3.1, ALS \\
Wavelength $(\AA)$ & 1.01 & 1.01 \\
Temperature $(\mathrm{K})$ & 100 & 100 \\
Detector & PILATUS3 S 6M & PILATUS3 S 6M \\
Space group & $P 2_{1}$ & $P 2_{1}$ \\
$a, b, c(\AA)$ & $37.4,85.2,41.4$ & $37.3,85.1,41.3$ \\
$\alpha, \beta, \gamma\left({ }^{\circ}\right)$ & $90.0,106.2,90.0$ & $90.0,107.0,90.0$ \\
Mosaicity $\left({ }^{\circ}\right)$ & 0.20 & 0.55 \\
Resolution range $(\AA)$ & $35.90-1.35$ & $42.57-1.40$ \\
Total No. of reflections & 167120 & 134137 \\
No. of unique reflections & 52792 & 41849 \\
Completeness $(\%)$ & $96.7(86.6)$ & $86.3(81.0)$ \\
Multiplicity & $3.1(2.4)$ & $3.0(2.0)$ \\
$\langle I / \sigma(I)\rangle$ & $23.8(5.8)$ & $15.1(4.1)$ \\
$R_{\text {merge }}$ & $0.031(0.069)$ & $0.042(0.125)$ \\
$R_{\text {p.i.m. }}$ & $0.021(0.047)$ & $0.025(0.105)$ \\
CC ${ }_{1 / 2}$ & $0.998(0.995)$ & $0.998(0.990)$ \\
Overall $B$ factor from $_{\quad \text { Wilson plot }\left(\AA^{2}\right)}^{13.6}$ & & 13.9 \\
\hline
\end{tabular}

Table 3

Structure refinement.

Values in parentheses are for the highest resolution shell.

\begin{tabular}{|c|c|c|}
\hline Structure & $\begin{array}{l}\text { EcFtsZ(L178E)-GDP } \\
\text { (PDB code 6umk) }\end{array}$ & $\begin{array}{l}\text { EcFtsZ(L178E)-GTP } \\
\text { (PDB code 6unx) }\end{array}$ \\
\hline Resolution range $(\AA)$ & $35.90-1.35$ & $42.57-1.40$ \\
\hline Final $R_{\text {cryst }}$ & 0.186 & 0.185 \\
\hline Final $R_{\text {free }}$ & 0.204 & 0.205 \\
\hline \multicolumn{3}{|l|}{ No. of non-H atoms } \\
\hline Protein & 2176 & 2198 \\
\hline Ligand & 28 & 32 \\
\hline Water & 303 & 304 \\
\hline \multicolumn{3}{|l|}{ R.m.s. deviations } \\
\hline Bonds $(\AA)$ & 0.98 & 1.02 \\
\hline Angles $\left({ }^{\circ}\right)$ & 0.007 & 0.007 \\
\hline \multicolumn{3}{|l|}{ Average $B$ factors $\left(\AA^{2}\right)$} \\
\hline Protein & 23.9 & 25.4 \\
\hline Ligand & 18.3 & 24.1 \\
\hline Solvent & 33.3 & 34.5 \\
\hline \multicolumn{3}{|l|}{ Ramachandran plot } \\
\hline Favored regions (\%) & 99.0 & 98.3 \\
\hline Allowed (\%) & 1.0 & 1.7 \\
\hline Disallowed (\%) & 0.0 & 0.0 \\
\hline
\end{tabular}

data to $1.35 \AA$ resolution. As the $P$. aeruginosa FtsZ protein is the most similar in amino-acid sequence to EcFtsZ (overall identity of $67 \%$ over the conserved core of our truncated protein), we used the $P$. aeruginosa FtsZ-GDP structure (PDB entry 2vaw; Oliva et al., 2007) as a search model for molecular replacement. One clear solution was obtained, consistent with the presence of one EcFtsZ(L178E) molecule in the crystallographic asymmetric unit. Clear electron density was observed in the structure for GDP (Figs. 1 and 2a). After the addition of the GDP and water molecules and further refinement, the structure converged to final $R_{\text {cryst }}$ and $R_{\text {free }}$ values of $18.6 \%$ and $20.4 \%$, respectively, to 1.35 A resolution.

The GTPase activity of FtsZ is largely dependent on polymerization, as the GTP-binding pocket at the 'top' of a subunit must make contact with the catalytic T7 loop residues at the 
bottom of an adjacent subunit in the protofilament to hydrolyze the bound GTP (Erickson, 1998; Scheffers et al., 2002). Because EcFtsZ(L178E) has been shown to be deficient in GTPase activity owing to its inability to form filaments (Du et al., 2015), we considered that it was likely that it would crystallize with GTP. Crystals were successfully obtained of the EcFtsZ(L178E)-GTP complex that were isomorphous with the EcFtsZ(L178E)-GDP crystals. The EcFtsZ(L178)-GTP structure was solved and refined to final $R_{\text {cryst }}$ and $R_{\text {free }}$ values of $18.5 \%$ and $20.5 \%$, respectively, to $1.40 \AA$ resolution. Notably, the electron density for the $\gamma$-phosphate moiety of the GTP in the EcFtsZ(L178E)-GTP structure was weak (Fig. $2 b$ ); the average $B$ factor for the atoms of the $\gamma$-phosphate is $63.1 \AA^{2}$ compared with $19.2 \AA^{2}$ for the remaining atoms of the GTP. These data suggest that this mutant may retain residual GTPase activity or that the $\gamma$-phosphate is disordered in the structure.

\subsection{E. coli FtsZ(L178E) crystallizes in the form of straight protofilaments}

In spite of evidence that the L178E mutation in EcFtsZ inhibits assembly, the crystals showed that EcFtsZ(L178E) assembled into straight protofilaments, as noted in the previous study of Du et al. (2015). However, comparison of the EcFtsZ(L178E) filament with the SaFtsZ filament reveals that the EcFtsZ filament is much looser (see below for the buried surface areas). While the residues in the EcFtsZ(L178E) loops from residues 63 to 70 and 168 to 178 make somewhat similar interactions with neighboring subunits to those observed in the SaFtsZ filament, they are more surface-exposed in the EcFtsZ(L178E) crystal filament and their relatively high $B$ factors (the average $B$ factors for residues 63-70 and residues 168-178 are 56.9 and $45.5 \AA^{2}$, respectively, compared with the overall average protein $B$ factor of $23.9 \AA^{2}$ ) indicate they are somewhat flexible. In addition, the electron density for these residues is sparse. Presumably, as the residue corresponding to Leu178 in SaFtsZ is wedged into a hydrophobic pocket of an adjacent subunit in the SaFtsZ filament, the similar burial of the Glu178 side chain into the hydrophobic pocket in the EcFtsZ(L178E) filament is predicted to be unfavorable. As a result, the EcFtsZ(L178E) protofilaments have a more open subunit interface. In this filament, Leu68 from the loop connecting S3 and $\mathrm{H} 3$ is inserted into a hydrophobic pocket composed of Ala11, the $\mathrm{C}^{\beta}$ atom of Asp96, Ile200 and Phe210 of the adjacent subunit (Fig. $3 b$ ). Leu68 has an elevated average $B$ factor (48.6 $\AA^{2}$ ) compared with the overall $B$ factor of $23.9 \AA^{2}$ for the protein, suggesting that the contact might be weak. In the EcFtsZ(L178E) loop connecting H6 and H7, which contains the L178E mutation, Arg174 makes electrostatic interactions with Glu233 and stacks with the Arg271 side chain from the adjacent subunit, and Leu172 from the loop makes weak van der Waals interactions with the side chain of Leu272 from the neighboring subunit (Fig. $3 b$ ). Next to this loop, Phe137 also makes contacts with Leu272 and also Met206 and Phe275 from the adjacent subunit (Fig. $3 b$ ). Other cross-contacts include electrostatic interactions between Lys141 and Arg142 and the neighboring subunit residues Asp209 and Asp212, respectively. Consistent with the finding that the EcFtsZ(L178E) filament has fewer contacts between subunits, the buried surface area (BSA) between subunits in the interface of the EcFtsZ(L178E) protofilament is only $720 \AA^{2}$, compared with the BSA of $\sim 1200 \AA^{2}$ observed in the snug interface between subunits in the SaFtsZ filament (Krissinel \& Henrick, 2007; Matsui et al., 2012; Fig. 3a). This is similar to the BSA of $740-810 \AA^{2}$ that Matsui and coworkers observed for SaFtsZ mutants that polymerized in the $\mathrm{R}$ form (Matsui et al., 2014).

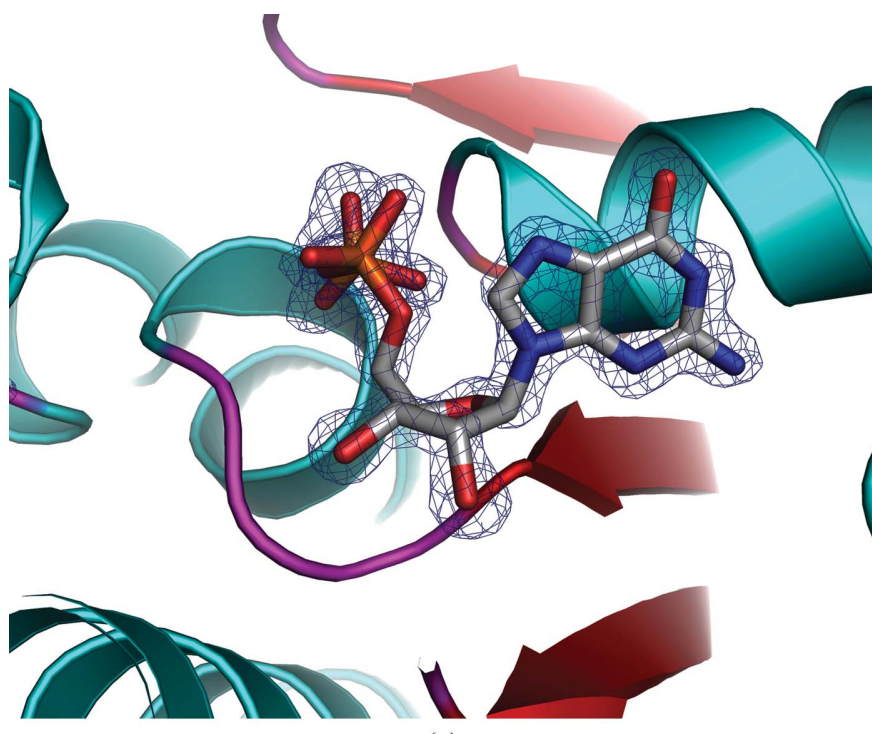

(a)

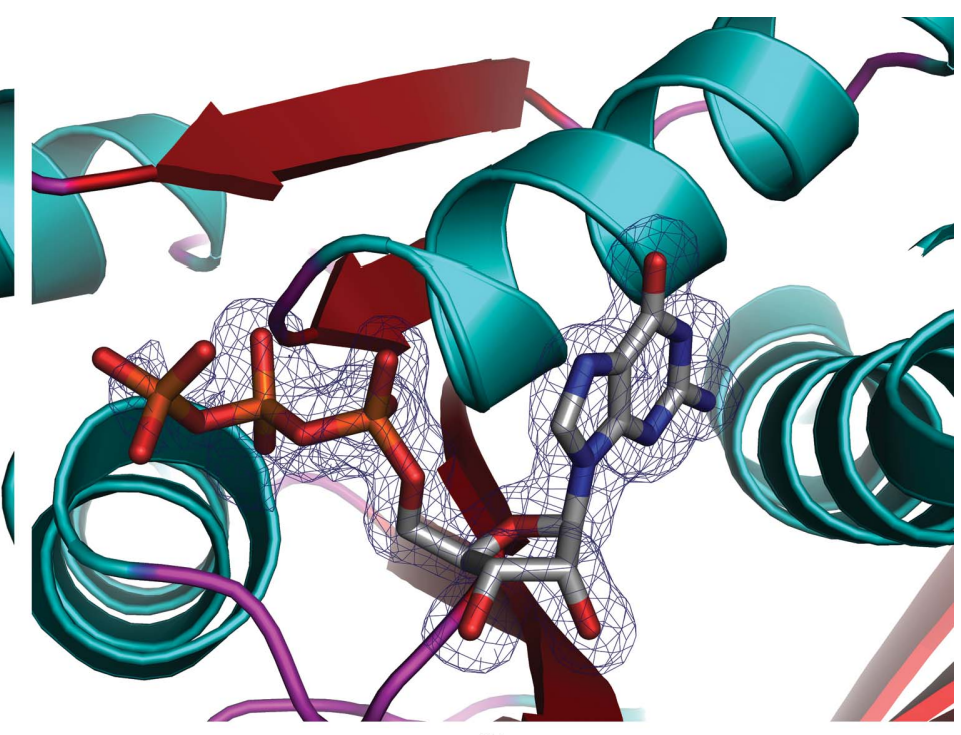

(b)

Figure 2

OMIT maps of GDP and GTP in EcFtsZ(L178E) structures. (a) Close-up of the GDP bound in the N-terminal subdomain pocket in EcFtsZ(L178E). The helices, strands and loops are colored cyan, red and magenta, respectively. The blue mesh corresponds to the $F_{\mathrm{o}}-F_{\mathrm{c}}$ OMIT map (in which the GDP was removed) contoured at $3.5 \sigma$ around the GDP. (b) Close-up of the GTP in the EcFtsZ(L178E)-GTP complex with the protein secondary-structural elements colored as in Fig. 2(a). The $F_{\mathrm{o}}-F_{\mathrm{c}}$ map is shown as a blue mesh and is contoured at $2.9 \sigma$. 
Further supporting the notion that the EcFtsZ(L178E) filament is not the form found in the Z-ring, the longitudinal spacing of subunits in the crystallized protofilament is $47.0 \AA$, which is significantly larger than the value of $42-43 \AA$ measured for EcFtsZ protofilaments by negative-stain EM (Erickson et al., 1996), the value of $44.0 \AA$ observed in the
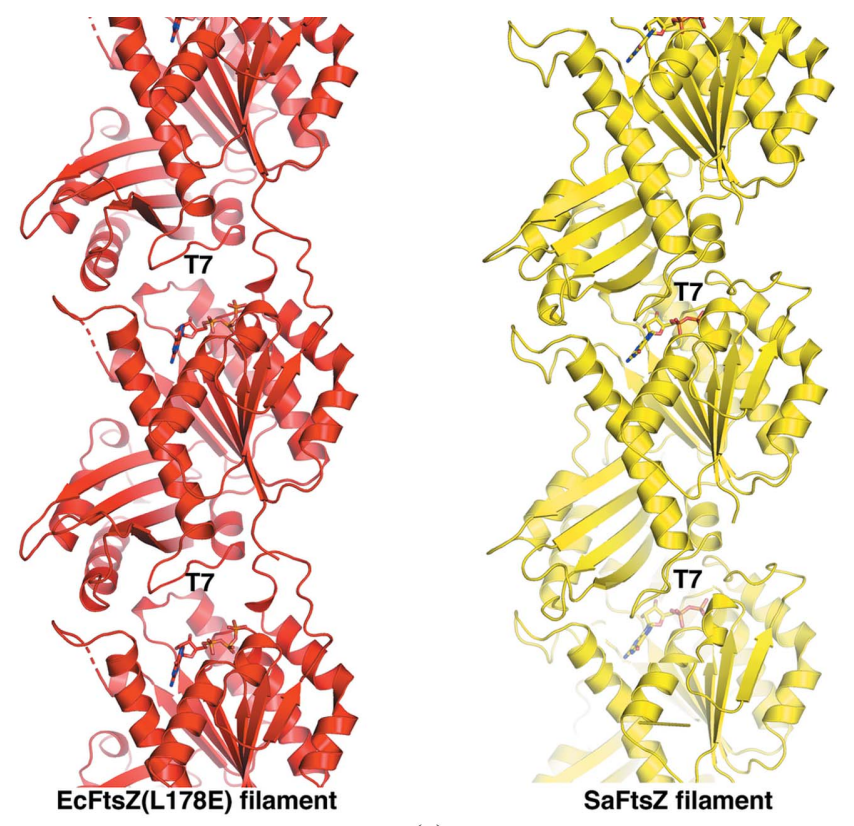

(a)

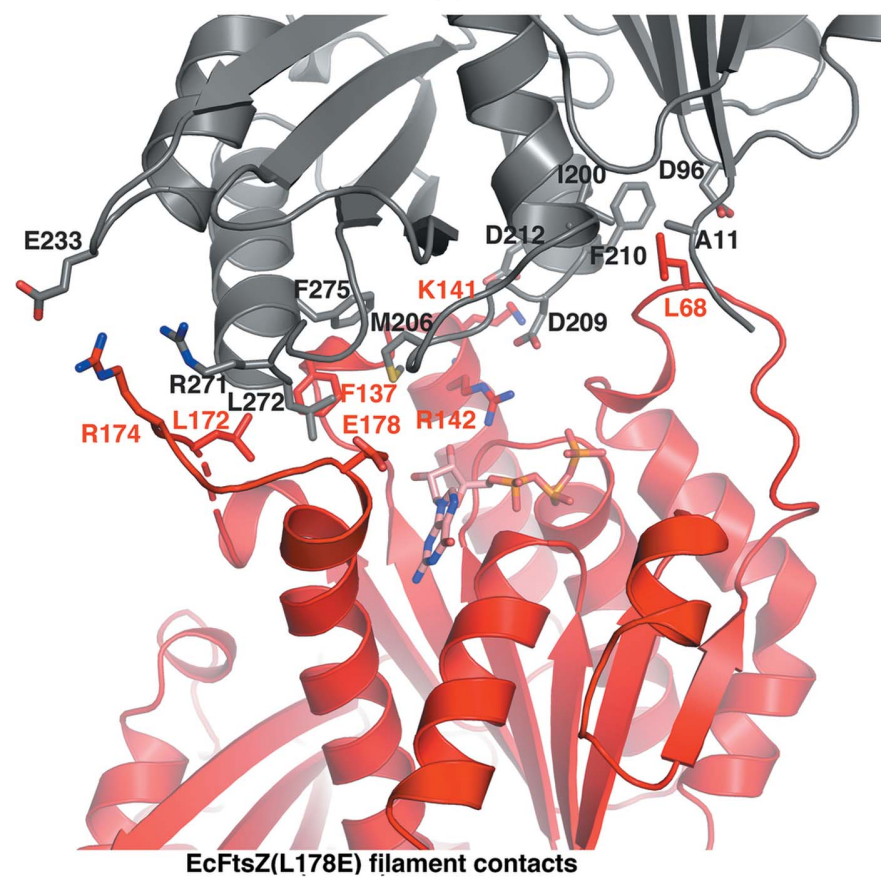

(b)

Figure 3

The EcFtsZ(L178E) filament. (a) Side-by-side comparison (after aligning the middle subunit) of the EcFtsZ(L178E) filament (red) generated by its crystal structure packing and the filament observed in the T-state SaFtsZ structure (PDB entry 3voa; yellow). The position of the T7 loop in each subunit is labeled, showing that this key catalytic loop is not properly positioned in the EcFtsZ filament (next to the GTP) as in the T-state filament. (b) Close-up view of the intersubunit contacts in the EcFtsZ(L178E) filament.
SaFtZ protofilaments in crystals (Matsui et al., 2012) and the value of $\sim 40 \AA$ measured by cryo-EM under native-like conditions (Szwedziak et al., 2014). Also in this filament, the T7 loops are not properly positioned to interact with the GTP of the subunit below (Fig. 3a). However, we note that this filament could represent an intermediate in the assembly or disassembly of functional filaments.

\subsection{E. coli FtsZ(L178E) structures are in the $\mathrm{R}$ conformation}

$D A L I$ structural homology searches revealed that the FtsZ structures with the strongest similarity to EcFtsZ(L178E) are the $P$. aeruginosa FtsZ (PDB entry 1ofu; Cordell et al., 2003) and S. aureus FtsZ (PDB entry 3wgl; Matsui et al., 2014) structures. PDB entry 1 ofu is the structure of $P$. aeruginosa FtsZ bound to SulA (Cordell et al., 2003), and 293 of the $\mathrm{C}^{\alpha}$ atoms in the FtsZ subunit of this structure could be superimposed onto the corresponding atoms in the EcFtsZ(L178E) structure with an r.m.s.d. of $1.1 \AA$ (Fig. 4a). The structure with PDB code $3 \mathrm{wgl}$ contains an $S$. aureus mutant in which the SGEVN sequence of the T7 loop was replaced by a shorter GAN sequence (Matsui et al., 2014). This FtsZ mutant was crystallized with GDP to produce the structure deposited as PDB entry 3 wgl. Despite the fact that $S$. aureus FtsZ shows $14 \%$ less sequence identity to the $E$. coli protein compared with $P$. aeruginosa FtsZ, PDB entry $3 \mathrm{wgl}$ shows essentially the same degree of structural homology; $289 \mathrm{C}^{\alpha}$ atoms of the $3 \mathrm{wgl}$ $S$. aureus FtsZ structure could be superimposed onto the corresponding $\mathrm{C}^{\alpha}$ atoms of the $\mathrm{EcFtsZ}(\mathrm{L} 178 \mathrm{E})$ structure with an r.m.s.d. of $1.1 \AA$. Importantly, both PDB entries 1ofu and $3 \mathrm{wgl}$ are in the $\mathrm{R}$ conformation, supporting the adoption of the $R$ state by EcFtsZ(L178E) in the crystal. Indeed, superimposition of the EcFtsZ(L178E) structure onto the T-state conformation of FtsZ (PDB entry 3voa; Matsui et al., 2012) resulted in a large r.m.s.d. of $2.3 \AA$ for 269 corresponding $\mathrm{C}^{\alpha}$ atoms (Fig. 4b).

3.4. C-terminal subdomain structural differences revealed in filament compared with nonfilament FtsZ

Our analyses show that the EcFtsZ(L178E) structure adopts the $\mathrm{R}$ state and as such has a closed cleft between its $\mathrm{N}$-terminal and C-terminal subdomains (Wagstaff et al., 2017). When we compared the structure of the N-terminal subdomain of the EcFtsZ(L178E) structure with the N-terminal subdomain of both R-state and T-state FtsZ structures, we found they are all very similar; they all overlay with r.m.s.d.s of $<1 \AA$ (Fig. 4c). Interestingly, although the C-terminal subdomain of EcFtsZ(L178E) is structurally similar to those of other R-state structures (r.m.s.d.s of between 0.8 and $1.2 \AA$; Fig. 4d), T-state C-terminal subdomain structures showed significant structural differences from the EcFtsZ(L178E) C-terminal subdomain and thus other R-state C-terminal subdomains, with r.m.s.d.s of $>2.1 \AA$ for these overlays (Fig. 4e). A main difference in these $\mathrm{C}$-terminal subdomain structures appears to be in the region encompassing the $\mathrm{T} 7$ loop and helix H8. The relevance of this structural difference 


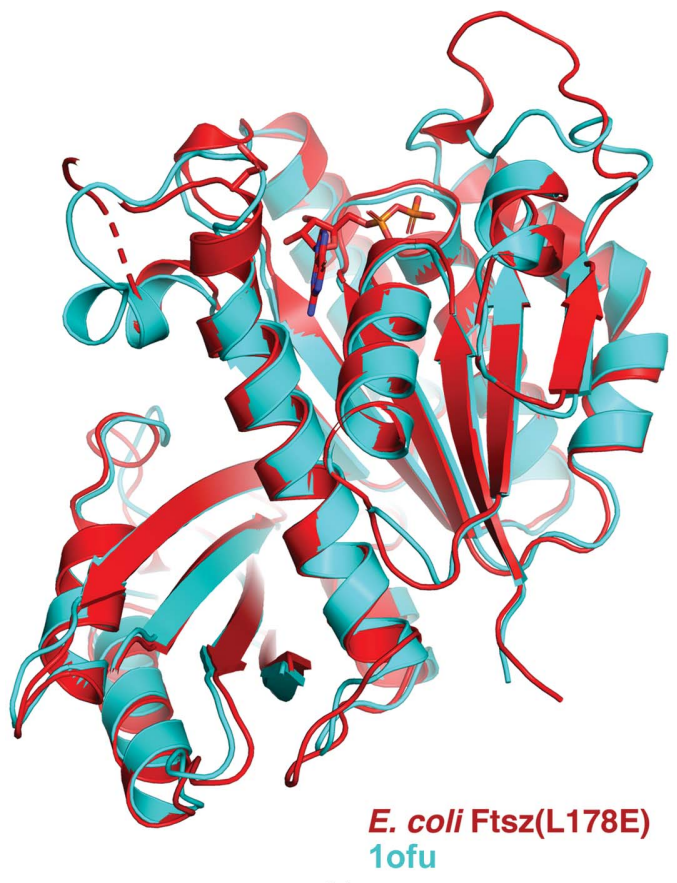

(a)

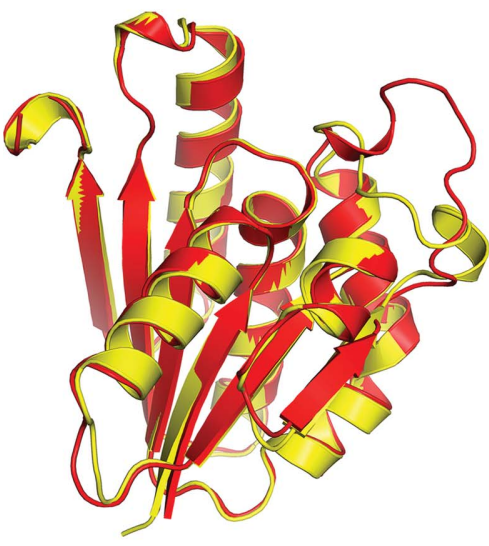

$\mathrm{N}$-domain overlays

(c)

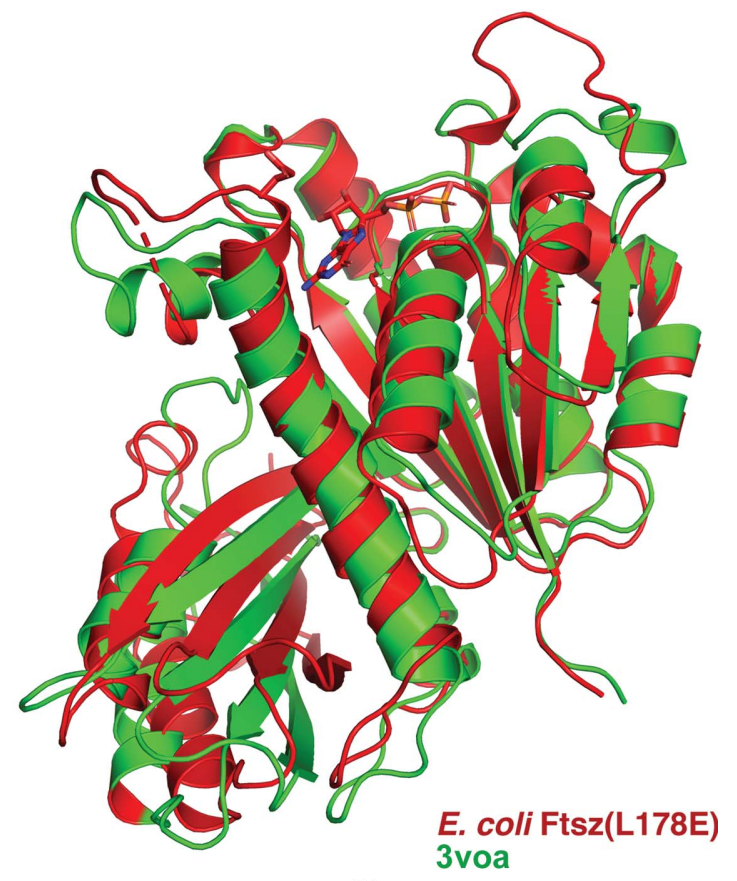

(b)

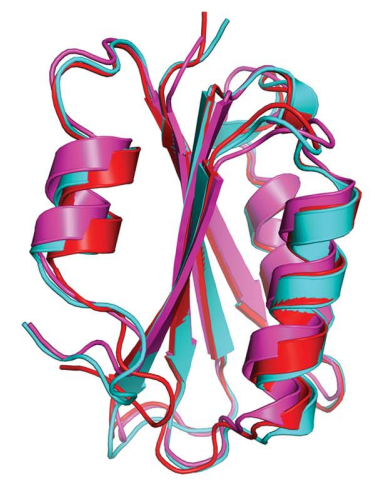

C-domain overlays/R state

(d)

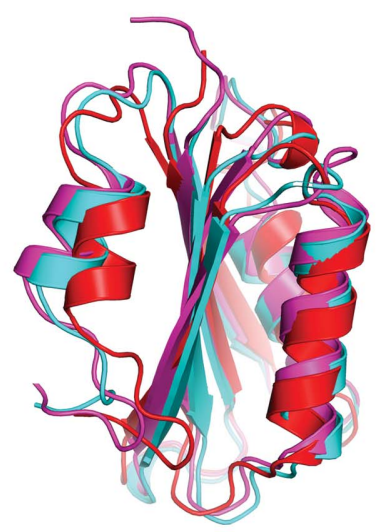

C-domains/R onto T states

(e)

Figure 4

The EcFtsZ(L178E) structure adopts the R state. (a) Superimposition of the EcFtsZ(L178E) structure (red) onto the R-state $S$. aureus FtsZ structure (PDB entry 1ofu; cyan). (b) Overlay of the EcFtsZ(L178E) structure (red) onto the T-state S. aureus FtsZ structure (PDB entry 3voa; green). (c) Overlay of the EcFtsZ(L178E) N-terminal subdomain (red) onto the N-terminal subdomain of the T-state structure (PDB entry 3voa; yellow), showing that the $\mathrm{N}$-terminal subdomains adopt the same conformations in R and T states. (d) Superimposition of the C-terminal subdomain of EcFtsZ(L178E) (red) onto the C-terminal subdomain of the R-state FtsZ structures (PDB entry 3wgl, magenta; PDB entry 1ofu, cyan), showing they have the same general fold (r.m.s.d.s of 1.1 and $0.8 \AA$, respectively). (e) Superimposition of the EcFtsZ(L178E) C-terminal subdomain (red) onto the C-terminal subdomains of the T-state FtsZ structures (PDB entry 4dxd, cyan; PDB entry 3voa, magenta), showing that the EcFtsZ (R-state) C-terminal subdomain has structural differences compared with T-state C-terminal subdomains (both r.m.s.d.s are $>2 \AA$ ).

between the C-terminal subdomains of the $\mathrm{T}$ and $\mathrm{R}$ states of FtsZ, if any, remains to be determined.

\section{Conclusions}

Studies in the last several decades using E. coli have revealed the machinery of the cell-division divisome and its association with the FtsZ Z-ring. A plethora of regulatory proteins that interact with $E$. coli FtsZ to affect its ability to form a Z-ring and its localization in the cell have also been discovered (Ortiz et al., 2016). However, to date a structure of EcFtsZ has not been reported. Here, we describe high-resolution structures of the EcFtsZ(L178E) protein in complex with GDP and GTP. These structures also allow the interpretation of multiple mutations that have been constructed and tested for their effects on cell division in this protein (Lu et al., 2001; Stricker \& Erickson, 2003; Addinall et al., 2005; Redick et al., 2005; Osawa et al., 2008; Haeusser et al., 2015; Márquez et al., 2017) and can serve as the foundation for the design of future celldivision experiments in $E$. coli to assess the role of specific residues in the function of FtsZ as well as its interactions with regulatory proteins. 


\section{Acknowledgements}

We thank the Advanced Light Source (ALS) and the support staff at beamline 8.3.1. Beamline 8.3.1 at the ALS is operated by the University of California Office of the President, Multicampus Research Programs and Initiatives grant MR-15328599, the National Institutes of Health (R01 GM124149 and P30 GM124169), Plexxicon Inc. and the Integrated Diffraction Analysis Technologies program of the US Department of Energy Office of Biological and Environmental Research. The Advanced Light Source, Berkeley, California is a national user facility operated by Lawrence Berkeley National Laboratory on behalf of the US Department of Energy under contract No. DE-AC02-05CH11231, Office of Basic Energy Sciences.

\section{Funding information}

This work was supported by a Nanaline H. Duke endowed chair and National Institutes of Health grants R35GM130290 (to MAS) and R01-GM066014 (to HPE).

\section{References}

Adams, D. W. \& Errington, J. (2009). Nat. Rev. Microbiol. 7, 642-653. Addinall, S. G., Small, E., Whitaker, D., Sturrock, S., Donachie, W. D. \& Khattar, M. M. (2005). J. Bacteriol. 187, 358-365.

Bi, E. \& Lutkenhaus, J. (1991). Nature, 354, 161-164.

Bisson-Filho, A. W., Hsu, Y.-P., Squyres, G. R., Kuru, E., Wu, F., Jukes, C., Sun, Y., Dekker, C., Holden, S., VanNieuwenhze, M. S., Brun, Y. W. \& Garner, E. C. (2017). Science, 355, 739-743.

Blaauwen, T. den, Hamoen, L. W. \& Levin, P. A. (2017). Curr. Opin. Microbiol. 36, 85-94.

Bramhill, D. \& Thompson, C. M. (1994). Proc. Natl Acad. Sci. USA, 91, 5813-5817.

Buske, P. J. \& Levin, P. A. (2013). Mol. Microbiol. 89, 249-263.

Cordell, S. C., Robinson, E. J. \& Löwe, J. (2003). Proc. Natl Acad. Sci. USA, 100, 7889-7894

Dajkovic, A. \& Lutkenhaus, J. (2006). J. Mol. Microbiol. Biotechnol. 11, 140-151.

Dajkovic, A., Mukherjee, A. \& Lutkenhaus, J. (2008). J. Bacteriol. 190, 2513-2526.

DeLano, W. L. (2002). PyMOL. http://www.pymol.org.

Du, S. \& Lutkenhaus, J. (2017). Mol. Microbiol. 105, 177-187.

Du, S. \& Lutkenhaus, J. (2019). Trends Microbiol. 27, 781-791.

Du, S., Park, K.-T. \& Lutkenhaus, J. (2015). Mol. Microbiol. 95, $173-$ 188.

Elsen, N. L., Lu, J., Parthasarathy, G., Reid, J. C., Sharma, S., Soisson, S. M. \& Lumb, K. J. (2012). J. Am. Chem. Soc. 134, 12342-12345. Erickson, H. P. (1998). Trends Cell Biol. 8, 133-137.

Erickson, H. P., Anderson, D. E. \& Osawa, M. (2010). Microbiol. Mol. Biol. Rev. 74, 504-528.

Erickson, H. P., Taylor, D. W., Taylor, K. A. \& Bramhill, D. (1996). Proc. Natl Acad. Sci. USA, 93, 519-523.

Fujita, J., Harada, R., Maeda, Saito, Y., Mizohata, E., Inoue, T., Shigeta, Y. \& Matsumura, H. (2017). J. Struct. Biol. 198, 65-73.

Gardner, K. A., Moore, D. A. \& Erickson, H. P. (2013). Mol. Microbiol. 89, 264-275.

Haeusser, D. P. \& Margolin, W. (2016). Nat. Rev. Microbiol. 14, 305319.

Haeusser, D. P., Rowlett, V. W. \& Margolin, W. (2015). Mol. Microbiol. 97, 988-1005.

Huecas, S., Llorca, O., Boskovic, J., Martín-Benito, J., Valpuesta, J. M. \& Andreu, J. M. (2008). Biophys. J. 94, 1796-1806.

Jones, T. A., Zou, J.-Y., Cowan, S. W. \& Kjeldgaard, M. (1991). Acta Cryst. A47, 110-119.
Krissinel, E. \& Henrick, K. (2007). J. Mol. Biol. 372, 774-797.

Leslie, A. G. W. (2006). Acta Cryst. D62, 48-57.

Leung, A. K. W., White, E. L., Ross, L. J. \& Borhani, D. W. (2000). Acta Cryst. D56, 1634-1637.

Li, Y., Hsin, J., Zhao, L., Cheng, Y., Shang, W., Huang, K. C., Wang, H.-W. \& Ye, S. (2013). Science, 341, 392-395.

Li, Y., Shao, S., Xu, X., Su, X., Sun, Y. \& Wei, S. (2018). ACS Nano, 12, 6137-6146.

Liebschner, D., Afonine, P. V., Baker, M. L., Bunkóczi, G., Chen, V. B., Croll, T. I., Hintze, B., Hung, L.-W., Jain, S., McCoy, A. J., Moriarty, N. W., Oeffner, R. D., Poon, B. K., Prisant, M. G., Read, R. J., Richardson, J. S., Richardson, D. C., Sammito, M. D., Sobolev, O. V., Stockwell, D. H., Terwilliger, T. C., Urzhumtsev, A. G., Videau, L. L., Williams, C. J. \& Adams, P. D. (2019). Acta Cryst. D75, 861-877.

Loose, M. \& Mitchison, T. J. (2014). Nat. Cell Biol. 16, 38-46.

Löwe, J. \& Amos, L. A. (1998). Nature, 391, 203-206.

Lu, C., Stricker, J. \& Erickson, H. P. (1998). Cell Motil. Cytoskeleton, 40, 71-86.

Lu, C., Stricker, J. \& Erickson, H. P. (2001). BMC Microbiol. 1, 7

Lutkenhaus, J., Pichoff, S. \& Du, S. (2017). Cytoskeleton, 69, 778-790.

Ma, X., Ehrhardt, D. W. \& Margolin, W. (1996). Proc. Natl Acad. Sci. USA, 93, 12998-13003.

Margolin, W. (2005). Nat. Rev. Mol. Cell Biol. 6, 862-871.

Márquez, I. F., Mateos-Gil, P., Shin, J. Y., Lagos, R., Monasterio, O. \& Vélez, M. (2017). Biochim. Biophys. Acta, 1859, 1815-1827.

Matsui, T., Han, X., Yu, J., Yao, M. \& Tanaka, I. (2014). J. Biol. Chem. 289, 3501-3509.

Matsui, T., Yamane, J., Mogi, N., Yamaguchi, H., Takemoto, H., Yao, M. \& Tanaka, I. (2012). Acta Cryst. D68, 1175-1188.

McCoy, A. J., Grosse-Kunstleve, R. W., Adams, P. D., Winn, M. D., Storoni, L. C. \& Read, R. J. (2007). J. Appl. Cryst. 40, 658-674.

Michie, K. A. \& Löwe, J. (2006). Annu. Rev. Biochem. 75, 467-492.

Miraldi, E. R., Thomas, P. J. \& Romberg, L. (2008). Biophys. J. 95, 2470-2486.

Mukherjee, A., Dai, K. \& Lutkenhaus, J. (1993). Proc. Natl Acad. Sci. USA, 90, 1053-1057.

Nogales, E., Downing, K. H., Amos, L. A. \& Löwe, J. (1998). Nat. Struct. Mol. Biol. 5, 451-458.

Oliva, M. A., Cordell, S. C. \& Löwe, J. (2004). Nat. Struct. Mol. Biol. 11, 1243-1250.

Oliva, M. A., Trambaiolo, D. \& Löwe, J. (2007). J. Mol. Biol. 373, 1229-1242.

Ortiz, C., Natale, P., Cueto, L. \& Vicente, M. (2016). FEMS Microbiol. Rev. 40, 57-67.

Osawa, M., Anderson, D. E. \& Erickson, H. P. (2008). Science, 320, 792-794.

Perez, A. J., Cesbron, Y., Shaw, S. L., Bazan Villicana, J., Tsui, H. T., Boersma, M. J., Ye, Z. A., Tovpeko, Y., Dekker, C., Holden, S. \& Winkler, M. E. (2019). Proc. Natl Acad. Sci. USA, 116, 3211-3220.

Pichoff, S. \& Lutkenhaus, J. (2005). Mol. Microbiol. 55, 1722-1734.

Potterton, E., Briggs, P., Turkenburg, M. \& Dodson, E. (2003). Acta Cryst. D59, 1131-1137.

Ramirez-Diaz, D. A., García-Soriano, D. A., Raso, A., Mücksch, J., Feingold, M., Rivas, G. \& Schwille, P. (2018). PLoS Biol. 16, e2004845.

Redick, S. D., Stricker, J., Briscoe, G. \& Erickson, H. P. (2005). J. Bacteriol. 187, 2727-2736.

Rowlett, V. W. \& Margolin, W. (2014). Biophys. J. 107, L17-L20.

Rowlett, V. W. \& Margolin, W. (2015). Philos. Trans. R. Soc. Lond. B Biol. Sci. 370, 20150028.

Scheffers, D.-J., de Wit, J. G., den Blaauwen, T. \& Driessen, A. J. M. (2002). Biochemistry, 41, 521-529.

Söderström, B., Chan, H. \& Daley, D. O. (2019). Curr. Genet. 65, 99101.

Söderström, B. \& Daley, D. O. (2017). Curr. Genet. 63, 161-164.

Strauss, M. P., Liew, A. T. F., Turnbull, L., Whitchurch, C. B., Monahan, L. G. \& Harry, E. J. (2012). PLoS Biol. 10, e1001389. 
Stricker, J. \& Erickson, H. P. (2003). J. Bacteriol. 185, 47964805.

Sundararajan, K., Miguel, A., Desmarais, S. M., Meier, E. L., Huang, K. C. \& Goley, E. D. (2015). Nat. Commun. 6, 7281.

Szwedziak, P., Wang, Q., Bharat, T. A., Tsim, M. \& Löwe, J. (2014). eLife, 3, e04601.

Tsang, M.-J. \& Bernhardt, T. G. (2015). Curr. Opin. Microbiol. 24,
$60-65$.

Vagin, A. \& Teplyakov, A. (2010). Acta Cryst. D66, 22-25.

Wagstaff, J. M., Tsim, M., Olivia, M. A., Garcia-Sanchez, A., Kureisaite-Ciziene, D., Andreu, J. \& Löwe, J. (2017). mBio, 8, e00254-17.

Yang, X., Lyu, Z., Miguel, A., McQuillen, R., Huang, K. C. \& Xiao, J. (2017). Science, 355, 744-747. 